\title{
Experiencia cubana en Cooperación Sur-Sur
}

\section{The Cuban experience in the South-South Cooperation}

\author{
Nestor Marimón Torres, ${ }^{\mathrm{I}}$ Evelyn Martínez Cruz $^{\mathrm{II}}$ \\ I Doctor en Medicina. Especialista de II Grado en Organización y Administración de \\ Salud Pública. Máster en Salud Pública. Ministerio de Salud Pública. La Habana, \\ Cuba. \\ II Doctor en Medicina. Especialista de I Grado en Medicina General Integral. Máster \\ en Salud Pública y Salud Internacional. Ministerio de Salud Pública. La Habana, \\ Cuba.
}

\section{RESUMEN}

Hoy se viven situaciones de crisis a nivel mundial pero la económica financiera es la que amenaza con serias repercusiones a nivel global sobre todos los aspectos del desarrollo de una nación, incluida la salud. Una de las peores consecuencias ya palpable es la reducción de la Ayuda Oficial al Desarrollo que proviene de las 22 naciones más ricas del mundo y se dirige hacia los países pobres y de bajos recursos. Se pretende profundizar en la importancia de la Cooperación Sur-Sur para enfrentar situaciones de crisis y dentro de ella, un buen ejemplo de la efectividad en este tipo de cooperación lo constituye Cuba. Esta cooperación se realiza mediante la colaboración triangulada entre países del sur, transferencia de tecnologías y ayuda médica, derivados del desarrollo del Sistema Nacional de Salud cubano, además de ser parte de la política exterior del país. Cuba es un ejemplo de ayuda solidaria y desinteresada que se desarrolla desde el año 1963 con buenos resultados y demuestra que la cooperación es una vía eficaz de solución a los problemas de salud de las poblaciones siempre y cuando se trate de una ayuda justa y solidaria, dirigida a fortalecer las capacidades nacionales y acorde con las prioridades del país que la recibe.

Palabras clave: Cooperación Sur-Sur, Ayuda Oficial al Desarrollo, crisis financiera, colaboración médica, solidaridad, recursos humanos en la salud, capacidades nacionales, Cuba. 


\begin{abstract}
The people are living today amid difficult situations caused by the world crisis; however, the financial-economic crisis is the one threatening the nations with serious global impacts, mainly in all aspects of development including health. One of the worst tangible consequences is the reduction of the Official Assistance for Development given by the 22 wealthiest countries to the poor and the resourcelimited nations. This paper was intended to delve into the importance of the southsouth cooperation to face crisis situations and a good example of the effectiveness of this type of collaboration is Cuba. This cooperation is based on the triangled collaboration among the southern countries, the transfer of technologies and the medical assistance derived from the development of the Cuban Health Care System, which is also part of its foreign policy. Cuba is an example of solidarity and unselfish assistance that has been put into practice since 1963 with good results. At the same time, it has proved that cooperation is an effective way of solving the health problems of the populations whenever this assistance is just and cooperative and aimed at strengthening the national capacities according to the priorities of the recipient country.
\end{abstract}

Key words: South-south cooperation, Official Assistance for Development, financial crisis, medical cooperation, solidarity, human resources in health care, national capacities, Cuba.

\title{
EL MUNDO, LA SALUD Y LA COOPERACIÓN INTERNACIONAL
}

En el mundo existen más de seis mil millones de seres humanos que lo habitan y viven momentos complejos que amenazan a la especie humana, dentro de ellos, las crisis, ya sea la económica, alimentaria, energética o medioambiental, consecuencia, entre otras razones, de las afectaciones producidas por el cambio climático; todas tienen serios efectos sobre la salud de las poblaciones, por lo que se requieren de consensos y de solidaridad para enfrentarlas y para buscar equidad y justicia social para todos.

Sin dudas la de mayor connotación es la económica financiera, todos los días se publican noticias sobre sus efectos negativos y realmente son alarmantes.

Ha sido calificada como la peor desde los años de 1930, se plantea que el crecimiento de la economía mundial será negativo, de menos de tres porciento, lo que conlleva a serias repercusiones e implicaciones sociales, por ejemplo: se estiman 239 millones de desempleados, es decir, 50 millones más en el mundo; la cifra de pobres llegará a 1422 millones, lo que representa un cuarto de la población mundial; sufrirán de hambre 1020 millones de personas, o sea, una sexta parte; estos son los datos más generales. ${ }^{1-3}$

Por supuesto, que las repercusiones de estas crisis se manifiestan a nivel local y hace que los gobiernos tomen medidas para sobrellevarlas, dentro de estas, los reajustes económicos y recortes de presupuestos; estas reducciones también incluyen al sector de la salud y se crea entonces un círculo vicioso, que se inicia con la reducción de las inversiones y presupuestos con lo que sufren mayoritariamente las poblaciones más vulnerables, que constituyen brechas dentro de los países, donde de por sí existen dificultades de acceso de todo tipo; aumenta el desempleo y el empleo informal, los niveles de pobreza, la desnutrición, la exclusión y las 
inequidades, se afecta a nivel global la Ayuda Oficial al Desarrollo (AOD) que realizan los países más ricos y que es de forma inestable en condiciones normalesy que repercute en el logro de Metas Globales de Salud como son los Objetivos de Desarrollo del Milenio, esto por ende tiene implicaciones en las economías de los países y continúa así este ciclo negativo y maligno empeorando la situación. ${ }^{4,5}$

Solo por citar algunos ejemplos de la grave situación mundial en salud, la diferencia entre la esperanza de vida entre los países ricos y pobres supera ya los 40 años, 10 millones de niños menores de 5 años mueren por causas evitables, cada $30 \mathrm{seg}$ muere un niño por paludismo, el $90 \%$ de ellos en África y cada 6 seg muere un niño por hambre. ${ }^{6}$

La OMS estima un déficit de 4,3 millones de médicos y otros trabajadores de la salud, fundamentalmente en el continente africano, que tiene, a su vez, una carga global de enfermedad del $24 \%$ pero solo recibe el $1 \%$ de financiamiento y cuenta con el $3 \%$ de trabajadores, mientras América solo tiene el $10 \%$ de carga de enfermedad, tiene el $50 \%$ del financiamiento y el $37 \%$ de trabajadores, sin embargo es el continente más inequitativo. Las diferencias son abismales y no se aprecia una estrategia organizada y dirigida a solucionar estos problemas. ${ }^{7,8}$

Ante esta grave situación, cabe una pregunta, ¿Cuál es la "salud" de la Cooperación Internacional?

Se entiende como AOD a todos los desembolsos netos de créditos y donaciones realizadas según los criterios de la Organización para la Cooperación y el Desarrollo Económico (OCDE), busca ayudar a los países más pobres mediante las políticas y préstamos de las instituciones de crédito del Sistema de Naciones Unidas.

En la 34 Sesión de la Asamblea General (1980), esta asistencia fue acordada al 0,70 \% del total del PIB. A pesar de este acuerdo, solo cinco países, de los 22 donantes cumplen con este compromiso: Noruega, Suecia, Luxemburgo, Dinamarca y Holanda. El resto de los países desarrollados ha mantenido su ayuda alrededor del $0,33 \% .{ }^{9,10}$

Al analizar la evolución de la AOD en América Latina y el Caribe en los últimos años, comprobamos que a fines de la década anterior alcanzó su nivel más bajo en términos reales. Tal situación contrasta con el lento pero sostenido crecimiento de la AOD destinada al resto de países en desarrollo. Algo distinto ocurrió en la primera mitad de la década de los noventa, periodo en el cual la AOD dispuesta para la región creció, en tanto se reducía la cantidad designada para los otros países en desarrollo (Fig.).

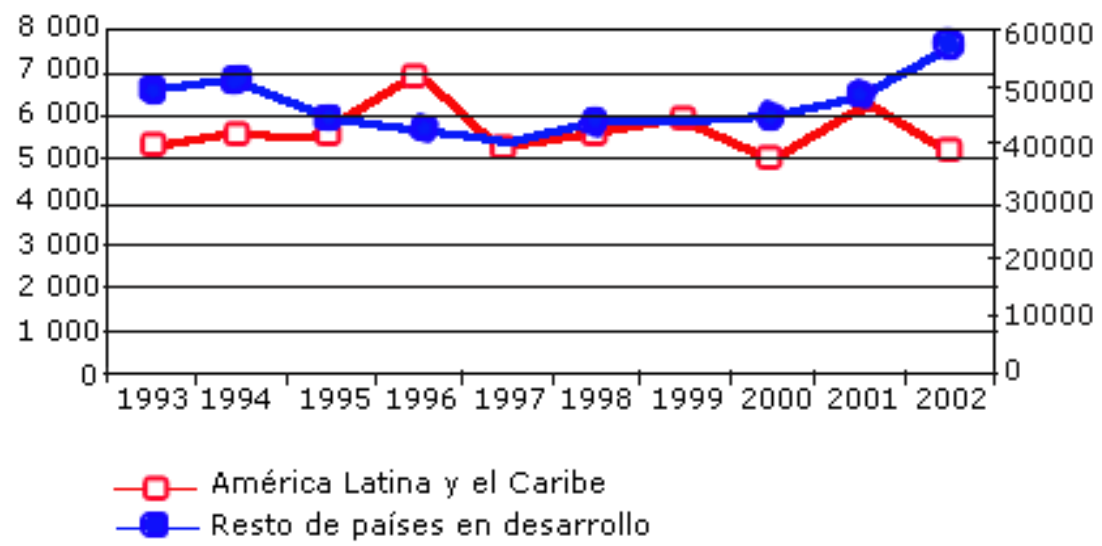

Nota: años 1993-2002 (USS millones a precios de 2002). Fuente: CAD/OCDE.

Fig. Desembolso neto de la ayuda oficial para el desarrollo para América Latina y el resto de los países en desarrollo 
Tras registrarse en 1996 el pico más alto de la AOD para América Latina y el Caribe, en la segunda mitad de los 90 la cifra disminuyó, tanto en cantidad como en importancia relativa; esto en comparación con la suma asignada a otras regiones, como África, Asia y Europa.

La razón esgrimida por los países cooperantes para disminuir la ayuda externa a América Latina ha sido la de considerar que la región tiene, en términos relativos, más ingresos que otras. Por tal motivo, no es considerada como prioritaria en la estrategia orientada a la lucha contra la extrema pobreza. Sin embargo, según las últimas estadísticas publicadas por la Comisión Económica para América Latina y el Caribe (CEPAL) en el año 2002, el $44 \%$ de la población de América Latina vivía en situación de pobreza y el $19,4 \%$ en la indigencia o pobreza extrema.

Asimismo, cabe señalar que la disminución de la AOD a la región no siempre ha servido para canalizar recursos para las regiones más pobres. Parte de esa ayuda se ha reorientado a zonas en conflicto bélico (como Irak o Afganistán) o, en el caso de la Unión Europea, a los países menos desarrollados de Europa.

En términos reales, el promedio anual de la AOD para América Latina y el Caribe durante la última década fue de US\$ 5623 millones, lo cual representa casi el $11 \%$ del total de la AOD destinada a los países en desarrollo.

En el año 2002, el monto de la AOD dirigido a la región fue de US\$ 5112 millones, el segundo más bajo en los últimos diez años. En ese mismo año, la región ocupaba el penúltimo lugar entre las regiones según el volumen total percibido de AOD, así como en término per cápita. ${ }^{11}$

En total los países desarrollados dan 106777 millones de USD anuales por concepto de AOD, mientras los países en desarrollo destinan 368 mil millones para el pago de la deuda, o sea, 3,4 veces más de lo que reciben, un balance negativo y absurdo. Esto ha sido llamado por algunos autores como la "Anticooperación."

El panorama mundial de actores de la cooperación internacional es ambiguo, por una parte se aprecia que la AOD es inestable con tendencia a la reducción y por otra, se produce un incremento de actores a nivel global, constituido por fondos, alianzas, ONGs, filantropías, esto hace que exista duplicidad de acciones en países que no tienen capacidades nacionales para canalizar esa ayuda, la misma se dirige hacia programas concretos y no está en concordancia con las prioridades de los ministerios de salud de los países, lo cual va en contra de la eficacia de la ayuda. Ante esta situación existe un llamado por la comunidad internacional: OMS, ministros de salud del Movimiento de Países No Alineados (MNOAL), informe de la comisión de Determinantes de Salud, sobre no reducir la ayuda en salud y que los países respeten la inversión en este sector (Marimón N. Martínez E. Conferencia presentada en el Global Fórum of Medica Research 2009. Cooperación Sur-Sur. Experiencia Cubana. Ciudad Habana, Noviembre 2009).

Mucho antes de que la situación empeorara de esta forma, ya Fidel hacía una alerta en este sentido, en el discurso pronunciado al celebrar el aniversario 45 del desembarco de los expedicionarios del Granma y el nacimiento de las Fuerzas Armadas Revolucionarias, en la Plaza "Antonio Maceo", de Santiago de Cuba, el 2 de diciembre de 2001, expresó:

El mundo será conquistado por las ideas y no por la fuerza, cuyo poder para sojuzgar y dominar a la humanidad será cada vez menor. Solo la paz y la cooperación entre los pueblos serán capaces de preservar a la humanidad de la muerte con que la amenazan por la vía del saqueo, la explotación, las guerras y la destrucción de las condiciones de vida del planeta. ${ }^{12}$ 


\section{LA EXPERIENCIA CUBANA DE COOPERACIÓN SUR-SUR}

Cuba tienen una experiencia de cooperación Sur-Sur de 49 años, la misma es parte de la política exterior del país y se rige por elementos claves o ejes, estos son:

- Se produce en beneficio e interés mutuo, equitativo entre los Estados. Cuba comparte lo que tiene.

- Es ofrecida sin ningún tipo de condicionamiento, con consenso nacional y voluntad política.

- Se desarrolla a partir de la suma de potencialidades de los países involucrados sin fines de lucro. Es una vía para la verdadera integración.

- Responde a las necesidades planteadas por los países receptores de la ayuda. Consiste en el envío gratuito de profesionales y técnicos de la salud principalmente dirigidos hacia la atención primaria de salud, en zonas rurales y remotas, con alto espíritu solidario y altruista.

- Los profesionales de la salud prestan sus servicios a toda la población, sin distinción de razas, credos o ideologías, sin mezclarse en asuntos políticos internos y respetando las leyes y costumbres (Marimón N. Martínez E. Conferencia presentada en el Global Fórum of Medica Research 2009. Cooperación Sur-Sur. Experiencia Cubana. Ciudad Habana, Noviembre 2009).

A pesar de ser un país pobre y necesitado, que además sufre desde el año 1960 un férreo bloqueo económico y comercial por EE. UU., que ya su costo económico se estima en 96 mil millones de USD y en la salud, de 2 mil 400 millones de USD, no ha dejado de brindar esta ayuda a lo largo de los años, que ha sido posible por las potencialidades del Sistema Nacional de Salud, el cual se rige por los siguientes principios:

1. La salud es un derecho de la población.

2. La salud de la población es responsabilidad del Estado.

3. Los servicios de salud alcanzan a toda la población por igual.

4. Las prácticas de salud tendrán una sólida base científica.

5. Las acciones de salud tendrán una orientación preventiva.

6. La participación social es inherente al manejo y desarrollo de los servicios de salud.

7. La solidaridad internacional será práctica de los servicios de salud. ${ }^{13}$ (Delgado García G. Centenario del Ministerio de Salud Pública de Cuba, 1909-2009. Coloquio por el centenario del Ministerio de Salud Pública de Cuba, Instituto Superior de Ciencias Médicas de La Habana, Ciudad Habana; enero 2009).

Cuenta además con el recurso más preciado: el capital humano, ya existen en el país un total de 566365 trabajadores de la salud, de estos, 74552 médicos y 32289 especialistas en Medicina General Integral para una proporción de un médico por 151 habitantes y 95,9 enfermeras por 10 mil habitantes.

Todo lo anterior ha sido posible por el desarrollo de las capacidades nacionales: 24 facultades de medicina, 499 policlínicos universitarios de la familia, 217 hospitales, 14007 consultorios médicos, 160 clínicas estomatológicas, 13 institutos de 
investigación con la red de instituciones del polo científico, todas trabajando con un solo objetivo; mejorar la salud de la población cubana. ${ }^{14}$

\section{Cuba y la solidaridad internacional}

La revolución cubana triunfa en 1959 y ese mismo año se produjo el éxodo masivo de médicos, de manera tal que el país perdió, en los primeros años de revolución, alrededor del $50 \%$ de los 6286 profesionales con que contaba. ${ }^{15}$

En 1960 cuando Chile fue afectado por un terremoto en el que perdieron la vida cientos de personas, se produce la primera ayuda con una brigada emergente, en este caso por un desastre natural.

Ya el 17 de Octubre del año 1962, se declara oficialmente el principio de la solidaridad internacional cuando Fidel, en el acto de inauguración del Instituto de Ciencias Básicas y Pre clínicas "Victoria de Girón", anuncia la decisión del gobierno cubano de brindar ayuda en el campo de la salud. Se considera entonces la fecha de inicio de la Colaboración Médica Internacional cubana con brigadas permanentes, el 23 de mayo de 1963, con el envío de la primera brigada a Argelia, compuesta por 55 colaboradores que brindan sus servicios por un año. ${ }^{16}$

Durante estos años, el número de colaboradores y de países donde se brinda la ayuda se incrementa, se gana en experiencia y se mejora cada día más en la calidad de la atención, se diversifica la cooperación médica en la mayoría de los campos de la salud y sobre todo en el área docente, tanto en Cuba como en el exterior a través de Brigadas Médicas.

Desde 1963 hasta diciembre de 2008 un total de 134849 colaboradores brindaron su ayuda en 108 países, agrupados por continentes de la siguiente forma:

- África: 38 países con 45488 participaciones.

- América: 39 países con 79717 participaciones.

- Europa, Asia-Pacífico y Medio Oriente: 31 países con 9644 participaciones.

Hoy existe colaboración medica en 76 países y 37820 trabajadores de la salud cumplen con esta responsabilidad (Informes de Archivos. 1998-2009.

Departamento Nacional de Estadísticas. MINSAP.)

\section{Desarrollo y evolución de la Colaboración Médica cubana}

Por supuesto que el desarrollo de la Colaboración Médica está en concordancia con la propia evolución del Sistema Nacional de Salud y marcada por el contexto nacional e internacional.

En el período de 1960-1980, el ámbito internacional estuvo caracterizado por los movimientos de liberación en África y Centroamérica, por lo que la ayuda se enfoca hacia estos países, se inicia la colaboración en la modalidad de misión internacionalista, la misma se basa en el principio de una ayuda solidaria gratuita. Ejemplos de países con colaboración en estos años fueron Argelia, Angola, Etiopía y Nicaragua.

http://scielo.sld.cu 
La década de los 90, estuvo marcada por eventos externos que afectaron sobremanera la economía cubana; dentro de ellos la desintegración de la URRS con la desaparición del campo socialista, nuestro principal mercado, el inicio del "período especial" y el recrudecimiento del bloqueo económico de EE. UU. hacia Cuba.

Existieron a la vez serios desastres naturales en Centroamérica que provocaron un viraje y redireccionamiento de la ayuda que se brindaba hasta esa fecha, momento en que aparece el Programa Integral de Salud (PIS), el 3 de noviembre de 1998, inicialmente en Centroamérica y el Caribe y posteriormente se extiende hacia África y el Pacífico y cuya esencia es el envío de Brigadas Médicas hacia lugares remotos, de difícil acceso, donde no había presencia de médicos nacionales.

Se crea en este período la Escuela Latinoamericana de Medicina, como elemento básico de continuidad y sostenibilidad del PIS.

A los integrantes de las Brigadas Médicas solo se les proporciona un dinero de bolsillo para las necesidades básicas. Hoy existen por esta modalidad un total de 4303 profesionales de la salud en todos los continentes, distribuidos de la siguiente forma: 2872 en América, 1176 en África y 255 en Asia y el Pacífico.

El nuevo milenio, con el impulso y desarrollo de la Revolución Bolivariana en Venezuela, se convierte en un factor externo que propicia la aparición de la modalidad de Programas Especiales, este fue Barrio Adentro el 16 de Abril de 2003, modalidad más avanzada y completa (en términos de servicios brindados dentro de un país), actualmente como expresión de la Alternativa Bolivariana para las Américas (ALBA) ${ }^{17}$

Otro importante suceso es la creación del Contingente Internacional de Médicos Especializados en Situaciones de Desastres y Graves Epidemias "Henry Reeve", el 25 de agosto de 2005 por iniciativa de Fidel, que se organiza como consecuencia de otro desastre natural; el huracán Katrina, que afectó a los territorios de Lousiana, Mississippi y Alabama en EE. UU. y constituye un nuevo enfoque de la Medicina de Desastre, aunque a partir de 1960 ya Cuba brindaba esta ayuda ante desastres naturales a través de Brigadas Emergentes. ${ }^{18}$

Las manifestaciones y posibilidades de ayuda se diversifican cada día, en el año 2004 se inició un importante programa: la Operación Milagro, el 8 de Julio de ese año llegan los primeros pacientes de Venezuela, el 22 de Julio de 2005 se ofrece a 15 países del Caribe y el 15 de septiembre de 2005 se extiende a 12 países de América Latina. ${ }^{19}$

La voluntad por garantizar la continuidad y el perfeccionamiento de las acciones realizadas hasta el momento, son los pilares básicos del éxito de esta hermosa tarea. Los países que reciben la ayuda reflejan mejores indicadores de salud que se pueden considerar como resultados de estos esfuerzos.

\section{Impactos y resultados de la cooperación cubana}

Dentro de los principales resultados que se aprecian durante todos estos años se encuentra el fortalecimiento de capacidades nacionales y la formación de recursos humanos en los países donde se brinda la ayuda, la apertura de 750 unidades o puestos de salud en la atención primaria, como esfuerzo para su fortalecimiento, atención a 92 hospitales comunitarios, cuatro instituciones sociales y un hogar de ancianos. Puesta en marcha de 14 hospitales y la apertura de 160 nuevos servicios hospitalarios, como refuerzo a las acciones realizadas en el primer nivel de atención.

http://scielo.sld.cu 
En los últimos 10 años de colaboración, las Brigadas Médicas cubanas han realizado más de 433 millones de consultas médicas, se han visto en terreno a 163840789 pacientes.

Se intervinieron quirúrgicamente 2695004 pacientes, se refuerza la infraestructura en salud con la creación de Centros Diagnósticos, ya suman 56 en total, distribuidos de la siguiente forma: 31 en Bolivia, 1 en Antigua y Barbudas, 1 en Haití, 1 en Sierra Leona y 1 en Dominica.

El Programa Especial de Cooperación con Venezuela, que se inició en abril de 2003, cuenta con 30685 profesionales cubanos de la salud, ostenta los siguientes resultados: 363084127 consultas, de estas 164210014 son visitas de terreno, 74398 operaciones, 6306 partos, 281892894 actividades educativas y 16538746 casos atendidos en ópticas.

Todos estos pacientes han sido atendidos en consultorios populares, Centros de Diagnóstico Integral que ya funcionan 500, Salas de Rehabilitación Integral con un total de 560 o en los 26 Centros de Alta Tecnología que ya existen. ${ }^{19}$ (Marimón N. Martínez E. Conferencia presentada en el Global Fórum of Medica Research 2009. Cooperación Sur-Sur. Experiencia Cubana. Ciudad Habana, Noviembre 2009).

\section{Ayuda ante situaciones de desastres}

A partir de 1960 un total de 45 Brigadas Médicas Emergentes han estado presentes en 30 países afectados por diversas catástrofes naturales: terremotos, huracanes, inundaciones, epidemias, erupciones volcánicas y otros.

La creación del Contingente Internacional de Médicos Especializados en Situaciones de Desastres y Graves Epidemias "Henry Reeve", constituye la institucionalización de la atención a través de la Medicina de Desastre en Cuba. Desde su creación han participado en 9 misiones, con un total de 4176 trabajadores de la salud, distribuidos entre en Guatemala, Pakistán, Bolivia, Indonesia, Perú, Belice, México, China y más recientemente El Salvador.

Como resultados se mencionan los siguientes: 3017718 consultas, 19199 operaciones, 991 partos y la donación de 36 hospitales de campaña dotados con equipos de la más alta tecnología.

\section{Programas de gran impacto social}

En la Operación Milagro, el principal resultado es el mejoramiento o devolución de la visión a 1825274 personas de 33 países. En Cuba se han operado 175610 pacientes y 1649664 en 60 centro oftalmológicos instalados en 18 países, con 93 puntos quirúrgicos donados por Cuba, con la más alta tecnología, en Venezuela, Bolivia, Ecuador, Guatemala, Haití, Honduras, Panamá, Nicaragua, Paraguay, Uruguay, Angola, Mali, Perú, Santa Lucía, San Vicente, Suriname, Guyana y Argentina. ${ }^{19}$ (Marimón N. Martínez E. Conferencia presentada en el Global Fórum of Medica Research 2009. Cooperación Sur-Sur. Experiencia Cubana. Ciudad Habana, Noviembre 2009).

Existe otro Programa importante y de gran impacto social que se desarrolló primero en Cuba y que ya se extiende a otros países, se trata del Estudio Psicopedagógico, Social y Clínico Genético de las personas con Discapacidad, se concluyó en Venezuela y se denominó Misión "Dr. José Gregorio Hernández", comprendió el periodo desde el 26 de julio de 2007 hasta el 26 de noviembre de 2008. 
Se estudiaron un total de 336270 personas con discapacidad, para una tasa de incidencia de 1,21 discapacitados por 100 habitantes. El estudio permitió evaluar desde el punto de vista social, psicológico y clínico genético a todas las personas con incapacidad, brindarles atención psicosocial y médica a todas las familias visitadas y cubrir las necesidades de ayudas técnicas y efectos electrodomésticos identificados.

El cúmulo de información arrojado por este estudio permitió a la dirección del país trazar políticas de salud, educación, cultura y contribuir de manera directa a disminuir la pobreza, así como el reconocimiento de la identidad de personas que vivían en el anonimato al no contar con los documentos correspondientes.

Como parte de la misión se instaló el primer laboratorio estatal de citogenética, primera parte de un complejo científico que se construirá en una instalación existente y que incluye un Centro de Genética Médica, Bioinformática y Neurociencias, instituciones que no existían en Venezuela. Por los resultados de esta investigación otros países solicitaron su realización y hoy se extiende a Nicaragua, Ecuador y Bolivia. ${ }^{20}$

\section{Cooperación triangulada}

Durante la Cumbre Sur-Sur del G 77, celebrada en el 2000 en La Habana, surge como acuerdo de los jefes de estado de Nigeria, Libia y Cuba, la creación de un Programa de Cooperación Sur-Sur como alternativa de cooperación en el campo de la salud entre los países del hemisferio sur. En este acuerdo Nigeria y Libia aportan el financiamiento y Cuba el recurso humano, es decir, la fuerza médica.

Los países que se identificaron para el desarrollo del programa fueron seis, todos del continente africano: Burkina Fasso, Níger, Chad, Sierra Leona, Mali y Gambia. Cuba aportó colaboradores en los cuatro primeros.

Durante los primeros cinco años del Programa de Cooperación, los logros en la asistencia prestada a la población de los países receptores incluyendo el apoyo en medicamentos, equipos médicos y ambulancias fue valorado en más de \$2 027000 USD. En sentido general, los resultados de esta cooperación son muy positivos ya que en el momento de su concepción era la única vía para que estos países, con muy pocos recursos económicos, pudieran contar con ayuda médica. En estos momentos Cuba mantiene su disposición de continuar colaborando con este tipo de Cooperación Sur-Sur. ${ }^{21}$

En el 2007, La OPS/OMS formula al Ministerio de Salud Pública de Cuba una propuesta de Cooperación Técnica Interregional entre las Oficinas Regionales de Salud de África y América de la Organización Mundial de la Salud (AMOR-AFRO), a desarrollarse en Angola, en el Marco de la Iniciativa Global de la Poliomielitis. El objetivo de la Cooperación es proporcionar asistencia técnica a las autoridades y técnicos de las provincias para alcanzar las metas de eliminación de la poliomielitis, vigilancia y control de otras enfermedades inmunoprevenibles, apoyar la planificación-implementación, el monitoreo y la evaluación de las actividades del Programa Alargado de Vacunaciones provinciales y municipales. Cuba aceptó y se enviaron a 20 especialistas cubanos a este país por un periodo de 6 meses.

Dentro de los principales resultados obtenidos se encuentran:

1. Vigilancia epidemiológica: se cumplió la lista exhaustiva y la clasificación de unidades sanitarias acordes al grado de prioridad y los avances en la ubicación de los puntos focales para la vigilancia activa de las comunidades.

http://scielo.sld.cu 
2. Vacunación de rutina: actualización de datos de cobertura y registros, avances en la calidad y frecuencia de las supervisiones.

3. Jornadas de vacunación: planificación y realización de campañas con calidad, acompañamiento y supervisión en el terreno.

4. Trabajo en equipo: reuniones de análisis mensual; punto focal OMS, consultor OMS, supervisores provinciales de vigilancia y del Programa de vacunación.

5. Capacitaciones: para personal del Ministerio de Salud, puntos focales, técnicos, representantes de la comunidad y población en general.

6. Investigación epidemiológica de los casos de poliomielitis diagnosticados.

7. Confección de Boletín Epidemiológico para los directivos provinciales.

Pero el principal resultado fue el logro de un trabajo en equipo entre asesores cubanos y supervisores del Programa de Vacunación angolano y puntos focales OMS.

Esta experiencia tuvo reconocimiento internacional: Margaret Chan, Directora General de la OMS, el 21 de mayo de 2008 en la I Reunión de Ministros de Salud del MNOAL, celebrada en el marco de la 61 Asamblea de la OMS, reconoce el apoyo de Cuba en la estrategia de erradicación de la poliomielitis.

David Heymann, asistente del Director General para la Seguridad de la Salud y el Medioambiente y representante del Director General para la Erradicación de la Poliomielitis, reconoció en este mismo marco, el apoyo de Cuba en el fortalecimiento de las capacidades nacionales en Angola y de la colaboración en general.

Mirta Roses, directora de la Oficina Regional para las Américas (OPS), realiza Conferencia en la sede de la OMS con el tema: Fomentando la Cooperación Técnica entre países: Experiencia OPS/AMOR, y pone de ejemplo esta Cooperación Sur-Sur que también involucra a organismos del sistema de Naciones Unidas. ${ }^{22}$ (Marimón T, Martínez E. Conferencia: Aporte de Cuba a la estrategia de erradicación de la Polio en Angola, un ejemplo de la Cooperación Técnica entre países. Congreso Internacional 70 Aniversario del IPK, Ciudad Habana, Cuba. Junio 2009).

Otro importante ejemplo en este tipo de ayuda, es la transferencia de tecnología que Cuba realiza al poner a disposición de los países del sur los avances científicotécnico alcanzados en la esfera de la biotecnología y que desarrollan las instituciones del Polo Científico. Con ese proceder se benefician terceros países y se potencian acciones conjuntas. Una importante operación en este sentido es la producción de vacuna Antimeningocócica AC mediante un intercambio entre el Instituto Finlay, en la Habana, Cuba y el de Tecnología e Inmunología de BioManguinhos en Río de Janeiro, Brasil, como respuesta a la solicitud de la OMS para cubrir el déficit de esta vacuna frente a la disminución de su producción por las trasnacionales farmacéuticas. Esta es una importante colaboración Sur-Sur, ayuda a los 21 países que comprenden el Cinturón de la Meningitis en África. ${ }^{23}$

\section{La formación de recursos humanos como parte de la Cooperación Sur-Sur}

El fortalecimiento de capacidades nacionales es una de las principales líneas de trabajo de la ayuda cubana, con la intención de aliviar la crisis de recursos humanos en el sector de la salud que existe a nivel internacional y de lograr la sostenibilidad y continuidad del trabajo de las Brigadas Médicas en el exterior. 
Desde 1963 hasta el 2008 se han graduado en Cuba un total de 11811 médicos procedentes de otros países.

En 1975 se creó la primera facultad de medicina en el exterior, con profesores cubanos en la ciudad de Aden en Yemen, y ya existen en Guyana, Etiopia, Guinea Bissau, Uganda, Ghana, Gambia, Guinea Ecuatorial, Haití y Eritrea.

En el curso escolar 2009-2010 hubo una matrícula de 51648 estudiantes en Cuba y en el exterior, los que se formaron junto a las Brigadas Médicas en los países, distribuidos de la siguiente forma: 8170 estudiantes en la Escuela Latinoamericana de Medicina, 12017 en el Nuevo Programa de Formación de Medicina, 1118 en otros proyectos, 29171 junto a las Brigadas y 1172 estudiando carreras técnicas.

La Escuela Latinoamericana de Medicina, creada el 15 de Noviembre de 1999 por idea de Fidel, también marcó un viraje en la concepción de la ayuda cubana en la formación de recursos humanos, inicialmente concebida para formar estudiantes de zonas remotas y postergadas del continente. Es una contribución de Cuba para ayudar a los países a saldar su propia deuda social, es un orgullo y ha graduado 7256 médicos de 30 países, cuenta actualmente con una matrícula de 8170 estudiantes de 28 países.

La formación de estudiantes en Medicina Integral Comunitaria en Venezuela, es otro importante proyecto y aporte para la sostenibilidad de acciones, tiene una matrícula de 28346 estudiantes, de ellos, 3643 en Premédica, 4262 en primer año, 4566 en segundo, 5697 en tercero y 10178 en cuarto año.

El Nuevo Programa de Formación de Médicos, que se aplica en Cuba desde la Atención Primaria de Salud y con la vinculación de estudio-trabajo, también se realiza con estudiantes extranjeros en el territorio nacional y fuera del país, con las Brigadas Médicas del Programa Integral de Salud, existen en esta modalidad 825 jóvenes que estudian en sus mismas comunidades y regiones, lo que contribuye a lograr una mayor sensibilización y compromiso para enfrentar las migraciones de recursos humanos del sector de la salud, que es un problemas dentro de la crisis actual, como ejemplos podemos citar a: Guinea Ecuatorial con 125 jóvenes, Eritrea con matrícula de 194, Timor Leste: 168, Guinea Bissau con 139, Gambia con 159 y Tanzania/Zanzibar con 40.

Cuba también apoya la formación de recursos técnicos y de enfermería, existen en el país 351 jóvenes estudiantes de países miembros del CARICOM y en Dominica se inauguró una Escuela con profesores cubanos situada en el Hospital Princess Margaret de Roseau, reinaugurada por el Primer Ministro Roosevelt Skerrit en febrero de 2006 y que cuenta con una matrícula de 127 estudiantes.

La solidaridad internacional, la cooperación y el intercambio de experiencias son decisivos para una Cooperación Internacional solidaria y efectiva, donde cada país aporta su principal riqueza. Cuba aporta el recurso humano en la mayoría de los casos, en otros se realiza transferencia de tecnologías y de esta forma se trazan en conjunto estrategias efectivas que sirven para dirigir la ayuda hacia la potenciación de capacidades nacionales, mejorar la salud de las poblaciones y enfrentar las diferentes crisis que afectan al mundo. Permite la unidad entre los pueblos y la sostenibilidad de acciones. ${ }^{19}$ (Marimón N. Martínez E. Conferencia presentada en el Global Fórum of Medica Research 2009. Cooperación Sur-Sur. Experiencia Cubana. Ciudad Habana, Noviembre 2009). 


\section{CONSIDERACIONES FINALES}

El fortalecimiento de capacidades nacionales y la creación de infraestructura, fundamentalmente a través de la formación de recursos humanos, así como la creación de las bases necesarias para que los propios países formen sus recursos, es básico para el desarrollo de la Cooperación Sur-Sur.

Estimular la cooperación triangulada es fundamental para potenciar acciones, ya existen ejemplos positivos:

- Programa de Colaboración Sur-Sur, dentro del grupo de los 77. Entre Nigeria, Libia y Cuba.

- Programa de erradicación de la poliomielitis en Angola. Cuba-OPS/OMS-Angola.

- Cooperación en la producción de vacunas antimeningococica AC para el continente africano. Instituto Finlay de Cuba y Biomanguinhos en Brasil a solicitud de la OMS.

- Transferencia de tecnologías para la producción de vacunas y medicamentos. Cuba-India, China, Viet Nam, Brasil y Argelia.

La continuación del fortalecimiento de Programas Especiales de Cooperación tales como, la Operación Milagro y los estudios clínico genéticos a personas con discapacidad, son otras vías de cooperación hacia poblaciones vulnerables y necesitadas.

La Cooperación Internacional a través de los procesos de integración como la Alianza Bolivariana para las Américas y el CARICOM son otras formas para alcanzar una verdadera integración, justa y solidaria para los pueblos.

Hay que mantener y fortalecer la unidad asistencial, docente y de investigación en la cooperación cubana para trabajar con vistas al futuro en la solución definitiva de los principales problemas de salud.

\section{REFERENCIAS BIBLIOGRÁFICAS}

1. Martínez O. Crisis económica global. ¿Hasta cuándo? ¿Hasta dónde? [Internet]. La Habana: Granma digital; 27 Abr 2009 [citado 14 Ago 2011]. Disponible en: http://www.granma.cubaweb.cu/2009/04/27/interna/artic01.html

2. Riera L. Desempleo crece sin tregua en los EE. UU. [Internet]. La Habana: Granma digital; 2009 [citado 7 Abr 2009]. Disponible en: http://www.granma.cu/espanol/2009/abril/mier4/vacufinlay.html

3. González L. Retrocederá más la economía mundial, pronostica la ONU [Internet]. La Habana: Granma digital; 2009 [citado 9 May 2009]. Disponible en:

http://alexisrojas.blog.com.es/2009/05/28/retrocedera-mas-economia-mundialpronostica-onu-6192071/

4. Akerman M. Social Determinats of health: Is this an international Issue? Washington, D.C.: OPS; 2009. 
5. Entrevista exclusiva a Mirtha Roses, Directora de la Organización Panamericana de la Salud. Washington, D.C.: Fondo Fiducidiario España-PNUD; 2009.

6. Organización Panamericana de la Salud. La recesión mundial y los Objetivos de Desarrollo del Milenio. ¿De qué manera la crisis económica puede afectar el cumplimiento de los ODM? Washington, D.C.: OPS; 2009.

7. Organización Mundial de la Salud. Informe Mundial de la Salud. Trabajemos por la salud. Ginebra: OMS; 2007.

8. Organización Mundial de la Salud. Informe Mundial de la salud. La Atención Primaria de Salud: Más necesaria que nunca. Ginebra: OMS; 2008.

9. Wikipedia, la enciclopedia libre [Internet]. Ayuda Oficial al Desarrollo [citado 22 Dic 2009]. Disponible en: http://es.wikipedia.org/wiki/Ayuda oficial al desarrollo

10. Chinchetru L. Coordinadora ONG para el desarrollo [Internet]. La ayuda oficial al desarrollo disminuyó en el 2007 [citado 22 Dic 2009]. Disponible en: http://www.congde.org/.../089c3572efe2e5a588118a5fcef806bb.pdf

11. Comisión Económica para América Latina y el Caribe. Panorama social de América Latina 2002-2003. Santiago de Chile: CEPAL; 2003.

12. Castro Ruz F. Acto para conmemorar el aniversario 45 del desembarco de los expedicionarios del Granma y el nacimiento de las Fuerzas Armadas

Revolucionarias [Internet]. Santiago de Cuba: Consejo de Estado; 2001 [citado 22 Dic 2009]. Disponible en:

http://www.cuba.cu/gobierno/discursos/2001/esp/f021201e.html

13. Informe de Cuba sobre la Resolución $63 / 7$ de la Asamblea General de las Naciones Unidas. Necesidad de poner fin al bloqueo económico, comercial y financiero impuesto por Estados Unidos de América contra Cuba. La Habana: Ministerio de Relaciones Exteriores; 2009.

14. Ministerio de Salud Pública (MINSAP). Dirección Nacional de Estadísticas. Anuario estadístico MINSAP 2008, La Habana: MINSAP; 2009.

15. Delgado García G. Conferencias de Historia de la Salud Pública en Cuba. Cuaderno de Historia No. 81, 1996.

16. Castro Ruz F. Discurso pronunciado en el acto de apertura del Instituto de Ciencias Básicas y Pre clínicas "Victoria de Girón" el 17 de Octubre de 1962 [Internet]. La Habana: Gobierno Revolucionario;1962 [citado 16 Jul 2011]. Disponible en: http://www.cuba.cu/gobierno/discursos/1962/esp/f171062e.html

17. Marimón Torres N. La colaboración Médica Cubana en el siglo xxi: Una propuesta para la sostenibilidad en Guinea Bissau [tesis]. La Habana: Escuela Nacional de Salud Pública; 2006.

18. Castro Ruz F. Discurso pronunciado en el encuentro con las fuerzas medicas prometidas para apoyar al pueblo de Estados Unidos en regiones afectadas por huracán Katrina. 4 de septiembre 2005 [Internet]. La Habana: Palacio de Convenciones; 2005 [citado 16 Jul 2011]. Disponible en:

http://www.cuba.cu/gobierno/discursos/2005/esp/f040905e.html 
19. Cooperación de Cuba en el mundo. La Habana: Ministerio de Relaciones Exteriores, Viceministerio de Cooperación Internacional; 2009.

20. Ministerio de Salud Pública (MINSAP). Informe final del Impacto social de las Misiones sociales. Reunión de la Comisión Intergubernamental Cuba-Venezuela. La Habana: MINSAP; 2009.

21. Ministerio de Salud Pública (MINSAP). Informe sobre avances en el programa Sur-Sur. Reunión de jefes de Brigadas Médicas Cubanas. La Habana: MINSAP; 2009.

22. Montalvo G. Informe final de la misión de apoyo al Programa de Erradicación de la Poliomielitis en Angola. La Habana: Oficina Regional de la OPS/OMS en Cuba; 2008.

23. Riera L. La colaboración médica antes y después de triunfo de la revolución. Ejemplo de colaboración Sur-Sur. Cuba y Brasil suministran vacuna a países africanos, en emergencia por Meningitis [Internet]. La Habana: Granma digital; 2008 [citado 7 Jun 2008]. Disponible en:

http://www.granma.cu/espanol/2008/junio/mier4/vacufinlay.html

Recibido: 23 de diciembre de 2009.

Aprobado: 1 de febrero de 2010.

Nestor Marimón Torres. Relaciones Internacionales del Ministerio de Salud Pública. Calle 23 No. 177. EL Vedado 10400. La Habana, Cuba. Correos electrónicos: dirdri@informed.sld.cu, evemart@infomed.sld.cu 